\title{
Mass Spectra of Octanes
}

\author{
By Evelyn G. Bloom, Fred L. Mohler, J. H. Lengel, and C. Edward Wise
}

\begin{abstract}
Mass spectra of the 18 isomers of octane have been measured. The sensitivity at the maximum peaks in each spectrum relative to $n$-butane is computed as well as the total ionization relative to $n$-butane. Tables and plots of relative intensities in the mass spectra are given, and some correlations with structure are found. All isomers with a tertiary butyl radical have similar spectra. The other isomers tend to dissociate at carbon atoms with one or two side chains attached to them. This determines whether the loss of two or three carbon groups in dissociation is probable. Loss of four carbon groups is probable whenever the molecule ion can break in half in a single dissociation process.
\end{abstract}

\section{Introduction}

Mass spectra of the 18 isomers of octane have been obtained as part of a systematic survey of mass spectra of pure hydrocarbons. ${ }^{1}$ It is of interest to compare the spectra of these isomers and look for possible correlations between the structure of the molecules and the mass spectra. These octanes are the highest molecular weight saturated hydrocarbons for which pure samples of all the isomers are available.

Unpublished mass spectra of the octanes have also been computed by the Consolidated Engineering Corporation for users of their mass spectrometer. A paper on mass spectrometer analyses of some liquid hydrocarbon mixtures ${ }^{2}$ describes the practical problems of analysing mixtures containing octanes by means of these mass spectra.

\section{Experimental}

Mass spectra have been obtained on a $180^{\circ}$ Consolidated mass spectrometer equipped with automatic control of the electron current and automatic temperature control of the ionization chamber. Standard procedures have been followed, and these are given in detail in the API Catalog (see footnote 1). Mass spectra were measured with $50-$ and $70-\mathrm{v}$ ionization potential, and the values at $50 \mathrm{v}$ are quoted here.

${ }_{1}$ Catalog of mass spectral data, American Petroleum Institute, Research Project 44, National Bureau of Standards, Washington 25, D. C.

${ }^{2}$ R. A. Brown, R. C. Taylor, F. W. Melpolder, and W. S. Young. Anal. Chem. 20, 5 (1948)
Relative intensities of mass peaks are expressed in terms of the maximum peaks taken as 100 percent and include intensities down to 0.01 percent. The sensitivity at the maximum peaks is measured in terms of the scale divisions of galvanometer deflection per micron pressure in the gas reservoir. The sensitivity at the mass 43 peak of $n$-butane is recorded for each octane spectrum, and the ratio of sensitivity of an octane to that of $n$-butane is a molecular property nearly independent of the arbitrary units used. Details of the mass spectral data are given in the API mass spectral catalog (see footnote 1).

The compounds used were with one exception National Bureau of Standards standard samples of specified purities between 99.5 and 99.94 mole percent. Tetramethylbutane (the only solid octane) was a sample of better than 98 mole percent purity obtained from the Ethyl Corporation by API Research Project 6 .

\section{Results}

Sensitivity and total ionization.-Table 1 gives data on the sensitivity and total ionization of the octanes as compared with $n$-butane. Column 1 lists the 18 isomers in the order of increasing complexity of side chains. Column 2 gives the maximum mass peak in each spectrum, and in most cases it is mass 43 . Four isomers have the maximum at 57, and these are the isomers that have a tertiary butyl radical $\mathrm{C}\left(\mathrm{CH}_{3}\right)_{3}$, and in the case of 2 , 2,3,3-tetramethylbutane, both halves of the mole- 
cule have this structure. Evidently molecule ions containing this grouping have a high probability of splitting in half.

Column 3 gives values of the ratio of sensitivity at the maximum octane peak to the secsitivity at the $43^{+}$peak of $n$-butane. This ratio ranges in value from 1.40 to 2.76 with no obvious relation between the value and the molecular structure.

The sum of all the mass peaks in a spectrum multiplied by the sensitivity gives the total ionization in arbitrary units, and this number divided by the total ionization of $n$-butane is a molecular property nearly independent of instrumental factors. This ratio given in column 4 of table 1 , is 3.12 for $n$-octane and 1.86 for 2,2,3,3-tetramethylbutane with most other values intermediate. It is evident that total ionization depends on molecular structure though the correlation with structure is again not obvious.

Relative intensities.-Table 2 lists the relative peak heights of 17 mass peaks in the octane spectra
TABLE 1. Octane mass spectral sensitivity

\begin{tabular}{|c|c|c|c|}
\hline Compound & $\begin{array}{l}\text { Maxi- } \\
\text { mum } \\
\text { peak }\end{array}$ & $\begin{array}{c}\text { Sensi- } \\
\text { tivity at } \\
\text { maximum } \\
\text { peak } \\
\text { (relative } \\
\text { to } n- \\
\text { butanea) }\end{array}$ & $\begin{array}{c}\text { Total } \\
\text { ionization } \\
\text { (relative } \\
\text { to } n- \\
\text { butane) }\end{array}$ \\
\hline$n$-Octane $\ldots$ & 43 & 2.46 & 3.12 \\
\hline 2-Methylheptane & 43 & 1.87 & 2.52 \\
\hline 3-Methylheptane & 43 & 1. 60 & 2.42 \\
\hline 4-Methylheptane & 43 & 2.13 & 2. 29 \\
\hline 2,2-Dimethylhexane & 57 & 2. 76 & 2.14 \\
\hline 2,3-Dimethylhexane $\ldots$ & 43 & 1. 63 & 1.94 \\
\hline 2,4-Dimethylhexane & 43 & 1.42 & 2.07 \\
\hline 2,5-Dimethylhexane $\ldots$ & 43 & 1. 56 & 2.08 \\
\hline 3,3-Dimethylhexane ............ & 43 & 1.58 & 1.97 \\
\hline 3,4-Dimethylhexane & 56 & 1. 40 & 1.84 \\
\hline 3-Ethylhexane & 43 & 2.45 & 2.43 \\
\hline 2,2,3-Trimethylpentane & 57 & 2.24 & 2.09 \\
\hline $2,3,3$-Trimethylpentane $\ldots \ldots$ & 43 & 1.80 & 2.15 \\
\hline 2,3,4-Trimethylpentane & 43 & 1. 99 & 2.15 \\
\hline $2,2,4$-Trimethylpentane $\ldots \ldots \ldots$ & 57 & 2.53 & 2.03 \\
\hline 2-Methyl, 3-ethylpentane & 43 & 1. 98 & 2. 20 \\
\hline 3-Methyl, 3-ethylpentane & 43 & 2.14 & 2.25 \\
\hline $2,2,3,3-$ Tetramethylbutane & 57 & 2.44 & 1. 86 \\
\hline
\end{tabular}

a Corrected to $0.675 \mathrm{~A}$ magnet current from $0.535 \mathrm{~A}$ values.

TABLE 2. Mass spectra of octanes

\begin{tabular}{|c|c|c|c|c|c|c|c|c|c|c|c|c|c|c|c|c|c|c|}
\hline \multirow{2}{*}{\multicolumn{2}{|c|}{ Compound }} & \multicolumn{17}{|c|}{ Percentage of maximum peak at $m / e$ values indicated } \\
\hline & & 114 & 112 & 99 & 85 & 84 & 71 & 70 & 69 & 57 & 56 & 55 & 43 & 42 & 41 & 29 & 27 & 15 \\
\hline & n-Octane ....... & 6. 9 & 0.02 & 0.1 & 30.0 & 6. 0 & 23.5 & 12. 2 & 1.3 & 34. 2 & 18.1 & 10. 2 & 100 & 15.8 & 38.9 & 34.5 & 26. 2 & 2. 2 \\
\hline (2) & 2-Me heptane.. & 4.9 & .04 & 12.6 & 1.8 & 0.8 & 12.9 & 17. 2 & 1. 3 & 73.3 & 8.1 & 11.1 & 100 & 41.9 & 38.2 & 27.7 & 25.3 & 3.1 \\
\hline (3) & 3-Me heptane & 3.0 & .02 & 0.8 & 49.0 & 27.3 & 3.1 & 2.5 & 2.7 & 67.5 & 38.3 & 11. 2 & 100 & 7.9 & 45.9 & 40.7 & 27.8 & 2. 8 \\
\hline (4) & $2,4-\mathrm{Me}_{2}$ hexane & 1. 7 & ..... & 1.1 & 46.1 & 9.0 & 14. 9 & 9.8 & 4. 2 & 72.8 & 29.6 & 9.9 & 100 & 11.0 & 43.4 & 32.4 & 24. 2 & 3. 6 \\
\hline (5) & $2,5-\mathrm{Me}_{2}$ hexane & 3.8 & 2. 02 & 17.3 & 0.7 & 0.2 & 18.8 & 9.7 & 1.4 & 80.2 & 7. 6 & 10.9 & 100 & 34.0 & 38.2 & 21.1 & 23.3 & 4. 4 \\
\hline (6) & $3,4-\mathrm{Me}_{2}$ hexane & 2. 2 & ...... & 0.3 & 38.1 & 7. 3 & 1.5 & 1.9 & 3. 3 & 79.2 & 100 & 9.9 & 68.1 & 4.0 & 56.5 & 48. 0 & 24.5 & 2. 7 \\
\hline (7) & 3-Et hexane & 1.6 & ...... & .1 & 28.5 & 22.6 & 13.5 & 12.9 & 2.5 & 12.4 & 5.9 & 11.6 & 100 & 6.7 & 22.8 & 19.8 & 19.3 & 1. 7 \\
\hline (8) & 2-Me,3-Et pentane & 1. 3 & & .1 & 18.1 & 4.3 & 24.7 & 50.0 & 3.3 & 14.8 & 2. 9 & 17.9 & 100 & 14. 2 & 27.0 & 18. 7 & 19.2 & 2. 2 \\
\hline & 3-Me,3-Et pentane & 0.00 & -...... & 1.9 & 64.3 & 16.8 & 0.5 & 0.6 & 5.8 & 27.0 & 2. 6 & 8.0 & 100 & 2.4 & 25.0 & 21.2 & 17.5 & 1.7 \\
\hline$(10)$ & $2,2-\mathrm{Me}_{2}$ hexane $\ldots$ & .03 & . & 5.6 & 0.02 & 0.01 & .7 & .3 & 0.8 & 100 & 32.2 & 4. 6 & 15.9 & 1.8 & 26.3 & 18.6 & 11.3 & 2.4 \\
\hline (11) & $2,2,3-\mathrm{Me}_{3}$ pentane & .03 & 0.01 & 3. 0 & 2.9 & .2 & .4 & .5 & 1. 2 & 100 & 57.5 & 5.8 & 22.8 & 2.2 & 33.4 & 23.0 & 11.4 & 2.9 \\
\hline (12) & $2,2,4-\mathrm{Me}_{3}$ pentane & .02 & ( & 4. 7 & 0.01 & .05 & .8 & .2 & 0.5 & 100 & 32. 7 & 4. 0 & 23.1 & 2.0 & 27.4 & 15.4 & 10.7 & 3. 6 \\
\hline (13) & $2,2,3,3-\mathrm{Me}_{4}$ butane & .03 & & 6. 2 & .03 & .3 & .3 & .1 & 1.3 & 100 & 27.3 & 4.5 & 17.7 & 1.5 & 28.2 & 16. 2 & 7. 0 & 4. 7 \\
\hline (14) & $3,3-\mathrm{Me}_{2}$ hexane $\ldots$ & .01 & .01 & 5.1 & 36.1 & 7. 6 & 47.0 & 17.1 & 3.5 & 40.7 & 5. 3 & 11.9 & 100 & 3.7 & 29.4 & 22.8 & 20.8 & 3. 0 \\
\hline$(15)$ & $2,3,3-\mathrm{Me}_{3}$ pentane & .01 & $-\ldots$ & 3.5 & 25.0 & 3.5 & 45.3 & 35.5 & 3.5 & 35.8 & 1.5 & 15.7 & 100 & 5.4 & 28.6 & 16. 0 & 18. 2 & 3.2 \\
\hline (16) & 4-Me heptane ..... & 3.1 & .02 & 0.9 & 4.5 & 3.0 & 52.7 & 46.1 & 1.3 & 14.4 & 2. 4 & 15. 3 & 100 & 13.5 & 26.6 & 22.8 & 22.5 & 2.4 \\
\hline (17) & $2,3-\mathrm{Me}_{2}$ hexane & 1. 7 & ...... & .4 & 1.5 & 0.4 & 46.4 & 58.3 & 1. 6 & 16. 7 & 1. 6 & 19.8 & 100 & 18.9 & 28.6 & 19.0 & 21.6 & 2.9 \\
\hline & $2,3,4-\mathrm{Me}_{3}$ pentane & 0.3 & $\ldots$ & .2 & 0.2 & 1 & 61.9 & 40.6 & 0.9 & 16. 3 & 2. 0 & 17.1 & 100 & 7.4 & 24.5 & 13.6 & 18. 2 & 3.3 \\
\hline
\end{tabular}

and figures 1, 2, and 3 show graphically 15 of the mass spectra and include all peaks greater than 2 percent of the maximum peak. In table 2 the compounds are listed in an arbitrary order to bring together groups with similar mass spectra, and the compounds are numbered consecutively. These numbers are used in the three figures for convenience of cross reference. The figures include structural formulas that omit the hydrogen atoms. In mass computations it is to be remembered that the valence of the carbon atom is four, and the appropriate number of hydrogen atoms must be added. Rearrangement peaks are indicated by $R$.

The spectra of the first nine isomers of table 2 and figures 1 and 2 show a great variety of intensity distributions and are very sensitive to molec- 


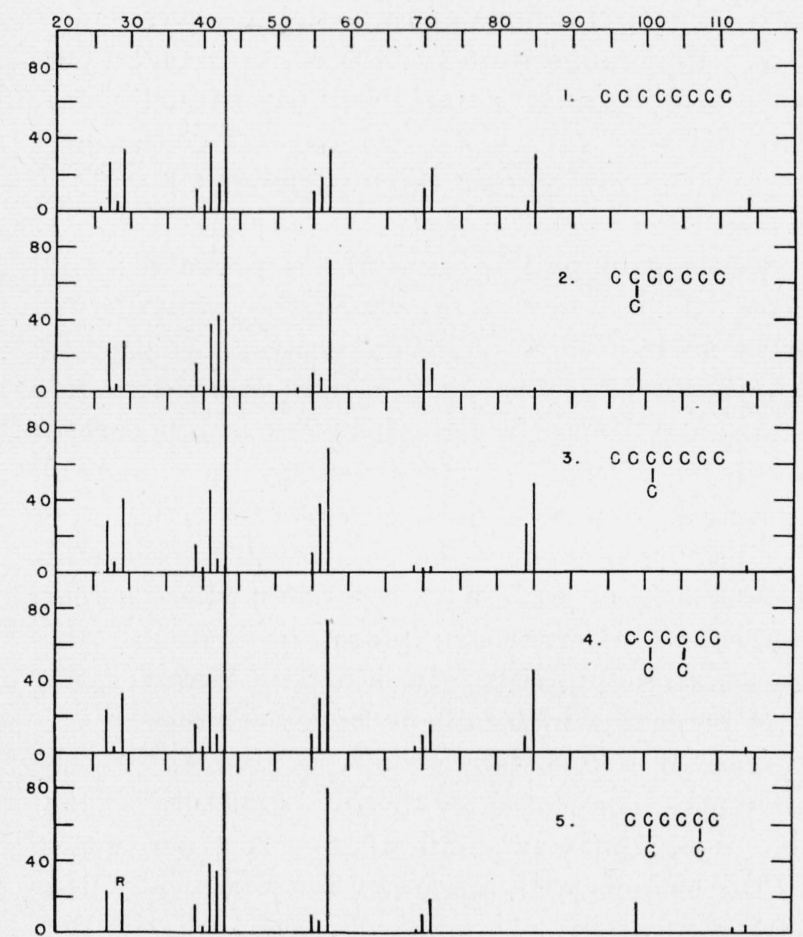

Figure 1. Mass spectra of octanes numbers 1 to 5 of table 2.

Abscissas are molecular weights, and ordinates are relative peak heights on a scale of 100 for the maximum peak. $R$ indicates a mass peak involving rearrangement of atoms before dissociation.

ular structure. The other nine can be divided into three groups that are similar within each group.

Isomers number 10 to 13 , inclusive, are the four compounds with a tertiary butyl radical which, as noted above, are characterized by having the maximum peak at 57 . It is seen in table 2 that these spectra are very similar with no large peaks of mass greater than 57. Only one of these, number 10 of figure 2 , has been plotted. If it is assumed that it is predominantly the $\mathrm{C}\left(\mathrm{CH}_{3}\right)_{3}$ half of the molecule that is ionized when the molecule ion dissociates, then the similarity of the four spectra is understood.

There is also a marked similarity between compounds 14 and 15 and between compounds 16,17 , and 18 shown in figure 3 . This is a consequence of some empirical relations between spectra and structure noted in the following paragraphs.

Mass spectra and structure.-The parent peak, $114^{+}$, has a maximum value for the normal compound, and this seems to be a general rule for saturated hydrocarbons. With a few exceptions the height of the peak decreases with increasing number of side chains. In table 2 it is seen that compounds number 9 to 15 , inclusive, have much smaller values of $114^{+}$than any other octanes. These values are 0.03 or less, and other values are 0.3 or greater. This group comprises all the octanes containing carbon atoms with two side chains.

Only one compound, 2,5-dimethylhexane, has an appreciable $112^{+}$peak. This peak is accompanied by a relatively large metastable transition peak at $110^{+}$, which comes from the delayed dissociation of $114^{+}$into $112^{+}$and two hydrogen atoms, after the parent ion has passed through the ion accelerating field. ${ }^{3}$

The $99^{+}$peak resulting from the loss of a $\mathrm{CH}_{3}$ radical from $114^{+}$has a wide range of values from 17.3 to 0.1 percent with no obvious relation between peak height and structure.

There seems to be a qualitative relation between

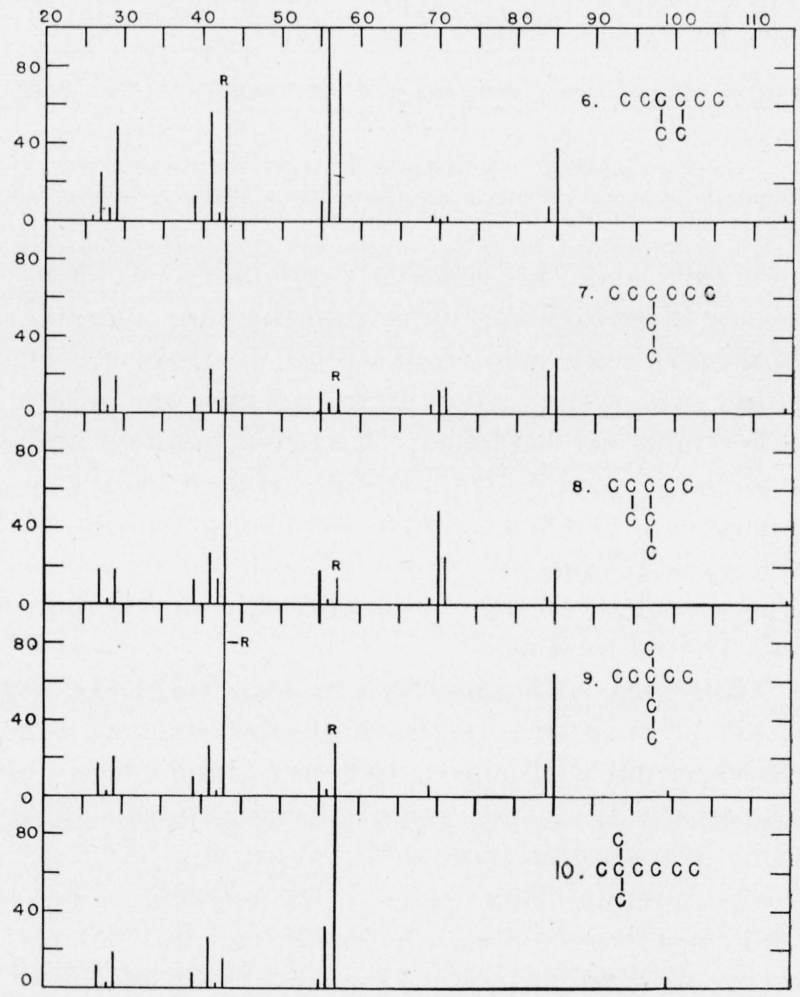

Figure 2. Mass spectra of octanes numbers 6 to 10 of table 2.

Abscissas are molecular weights, and ordinates are relative peak heights. $\mathrm{R}$ indicates mass peaks involving rearrangement of atoms before dissociation.

${ }_{3}$ Metastable transitions in mass spectra of 56 hydrocarbons, by Evelyn $\mathrm{G}$. Bloom, Fred L. Mohler, J. H. Lengel, and C. E. Wise, J. Research NBS 40 , 437 (1948) RP1888. 


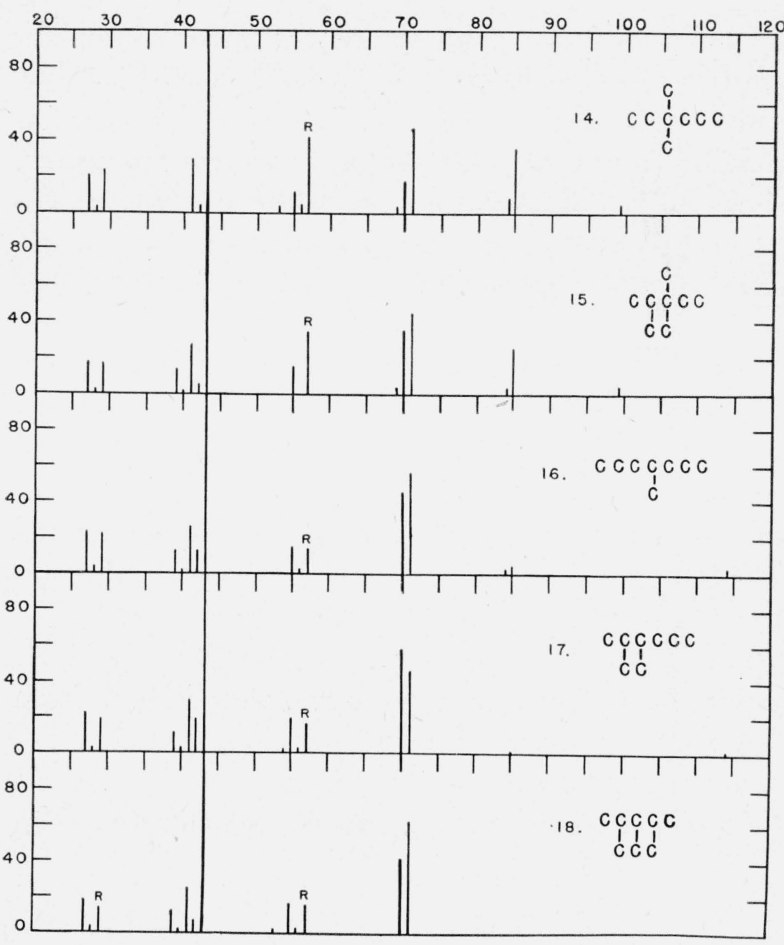

Figure 3. Mass spectra of octanes numbers 14 to 18 of table 2.

Abscissas are molecular weights and ordinates are relative peak heights. $\mathrm{R}$ indicates mass peaks involving rearrangement of atoms before dissociation.

structure and the relative probability of losing two or three carbon groups from the molecular ion. Molecules with side chains tend to dissociate on either side of the carbon atom to which one or two side chains are attached. The four isomers with a tertiary butyl radical can be excluded from consideration, as their spectra depend primarily on this radical and not on the rest of the structure. There remain 13 other isomers with one or more side chains to consider.

Molecules with an ethy] radical, $\mathrm{CH}_{3} \mathrm{CH}_{2}$, at either or both ends of the molecule attached to a carbon atom with one or two side chains tend to lose this radical and give a large $85^{+}$peak. The eight isomers that satisfy this condition have $85^{+}$ peaks ranging from 64.3 to 18 percent. Three that have ethyl radicals at either end but not attached to branch points have $85^{+}$peaks of 4.5 to 1.5 percent, and two that do not have ethyl radicals at the end have values of 0.7 and 0.2 percent.

Molecules with a propyl radical, $\mathrm{CH}_{3} \mathrm{CH}_{2} \mathrm{CH}_{2}$ or an isopropyl radical, $\left(\mathrm{CH}_{3}\right)_{2} \mathrm{CH}$ at the ends tend to lose these and give a large $71^{+}$peak. Ten isomers have propyl groups attached to branch points or have isopropyl groups at the ends and give values of $71^{+}$that range from 61.9 to 12.9 percent. One has a propyl group not attached to a branch point, and for this $71^{+}$is 3.1 percent. Two without terminal propyl groups have peaks of 1.5 and 0.5 percent.

Splitting in half to give $57^{+}$is probable in all cases except $n$-octane, in which the molecule can break in half in a single dissociation whether or not the break occurs at a carbon atom with side chains. As noted before this peak is 100 percent for the four isomers with a tertiary butyl radical. It ranges from 80.2 to 67.5 percent for the other five branched isomers that can break in half while it ranges from 40.7 to 12.4 percent where a rearrangement of hydrogen atoms or a double dissociation is required. In $n$-octane however, $57^{+}$ is 34 percent, which falls in the lower range.

Loss of a 5-carbon group to give $43^{+}$is not obviously correlated with the structure except that it is relatively small, 15.9 to 23.1 percent, in all the isomers with a tertiary butyl radical. It is 68.1 percent in 3,4-dimethylhexane and 100 percent in all the others. In 3-methyl, 3-ethylpentane, and 3,4-dimethylhexane this highly probable ionization process requires a rearrangement of atoms to give mass 43 .

The height of the $29^{+}$peak covers a smaller range of values and again is not obviously related to structure. In four cases a rearrangement of atoms is required to give $\mathrm{C}_{2} \mathrm{H}_{5}{ }^{+}$, these peaks tend to be smaller, but there are exceptions.

The $15^{+}$peaks cover a small range from 4.7 to 1.7 percent. 2,2,3,3-tetramethylbutane with six $\mathrm{CH}_{3}$ radicals has the largest $\mathrm{CH}_{3}{ }^{+}$peaks, but in general there is not a close correlation between the peak height and the number of $\mathrm{CH}_{3}$ radicals.

The similarity between 3,3-dimethylhexane and 2,3,3-trimethylpentane results from the above empirical rules. Both molecules can lose 2 carbon groups from one end or three trom the other end by breaking at a carbon atom with side chains, and neither molecule can break in half (see fig. 3). This makes $85^{+}$and $71^{+}$relatively large and $57^{+}$ small. Again in the last three isomers of table 2, the similarity comes from the fact that all three tend to lose three carbon groups but not two or four.

\section{Conclusions}

Chemical analysis. The great variety of octane spectra makes it easy to identify a nearly pure 
octane. To analyze a mixture of many octanes is necessarily difficult because of the large number of octanes. Also because of the variety of patterns, there is no basis for selecting an average pattern to analyze for octanes as a group when small amounts are mixed with other hydrocarbons.

To simplify analysis of a complicated mixture, one can use average patterns for the three groups of table 2: 10 to $13 ; 14$ and 15 ; and 16 to 18 . This reduces the number of unknowns in an octane mixture from 18 to 12 . In the absence of cyclics and olefines, the $112^{+}$peak is characteristic of 2,5-dimethylhexane and can be used to identify this if over 2 percent of the compound is present. The problem of analyzing for octanes can thus be reduced to 11 unknowns and 11 simultaneous equations. Electric computing machines capable of handling 12 unknowns are used in routine analyses ${ }^{4}$, so the problem could be solved by routine methods, but practical experience indicates that the precision would be low.

Brown, Taylor, Melpolder, and Young (see footnote 2) have made synthetic mixtures containing 16 of the octane isomers as well as $\mathrm{C}_{8}$

${ }^{4}$ C. E. Berry, D. E. Wilcox, S. M. Rock, and H. W. Washburn, J. Appl Phys. 17, 262 (1946). cyclics and have made use of the grouping described above. They find that for good precision of analysis, these mixtures have to be divided into three or more fractions by a fractionating column and the fractions analysed and summed.

Correlation of structure and spectra.-The possibility of deducing the structure of complicated molecules from the mass spectrum is still far from realization. A serious difficulty is that the phenomenon of rearrangement of hydrogen atoms that sometumes occurs in the process of ionization is not understood. This results in ions of masses that would not be expected from simple dissociation of the molecule. The empirical relations pointed out in the preceding section seem to apply with some generality to saturated hydrocarbons. However, they appear to be mutually inconsistent. Apparently in octanes, the high probability of ionizing a tertiary butyl radical almost completely masks the other possible ionization processes. Complications of this latter type are of course to be expected in molecules containing many atoms and many alternative steps in the dissociation process.

Washington, May 3, 1948. 